\title{
Tout empire
}

Ou comment ce concept a perdu sa spécificité et comment la restaurer

\section{Benoît Pélopidas}

\section{(2) OpenEdition}

\section{Journals}

Édition électronique

URL : http://journals.openedition.org/ress/855

DOI : $10.4000 /$ ress.855

ISSN : 1663-4446

Éditeur

Librairie Droz

\section{Édition imprimée}

Date de publication : 15 octobre 2011

Pagination : 111-133

ISBN : 978-2-600-01551-6

ISSN : 0048-8046

\section{Référence électronique}

Benoît Pélopidas, «Tout empire », Revue européenne des sciences sociales [En ligne], 49-1 | 2011, mis en ligne le 01 janvier 2015, consulté le 19 avril 2019. URL : http://journals.openedition.org/ress/855 ;

DOI : $10.4000 /$ ress. 855 


\title{
TOUT EMPIRE
}

\section{OU COMMENT CE CONCEPT A PERDU SA SPÉCIFICITÉ \\ ET COMMENT LA RESTAURER}

BENOÎT PELOPIDAS

Stanford University

bpelopidas@stanford.edu

\begin{abstract}
Résumé. Le débat intellectuel et politique récent a produit un étirement et une fragmentation du concept d'empire. Cet article poursuit donc trois objectifs: il cartographie les occurrences du terme d'empire afin d'identifier les concepts périphériques avec lesquels il est confondu, avance trois explications de cet étirement en étudiant les stratégies de définition du concept, puis propose une nouvelle définition. Pour ne pas ajouter à la confusion actuelle, elle devra distinguer l'empire des concepts périphériques d'hégémon, grande puissance, cosmopolis et impérialisme. En conclusion, nous revenons sur l'utilité d'un tel effort de clarification pour la science politique et la sociologie historique.
\end{abstract}

Mots-clés: empire; impérialisme; hégémon; cosmopolis; histoire des idées; théorie des relations internationales

\begin{abstract}
During the last few years, the concept of empire has suffered from conceptual stretching. The aim of this article is thus threefold. First, it identifies the peripheral concepts with which empire is usually confused. Second, it investigates defining strategies applied to "empire" and suggests three reasons that account for conceptual stretching. Third, it builds a new definition, carefully distinguished from those of hegemon, great power, cosmopolis and imperialism. Finally, the paper insists on the necessity of this clarification for political science as well as historical sociology.
\end{abstract}

Keywords: empire; imperialism; hegemon; cosmopolis; history of ideas; international relations theory 


\section{INTRODUCTION}

L'empire occupe une place ambiguë dans le débat politique et intellectuel contemporain. Le terme s'applique à un nombre croissant de formes politiques, voire économiques, et tend pour certains à désigner la forme politique majoritaire à une époque présente ou passée, voire l'époque elle-même, quand d’autres considèrent que cette forme de domination est révolue. Cet article entend prendre un peu de recul par rapport au foisonnement du débat pour comprendre les causes de la confusion régnante et contribuer à un effort de clarification. ${ }^{.}$Est-ce que l'empire est tout? Est-ce que tout est un empire? La question d'Alexander Motyl (2006) semble plus que jamais salutaire.

Après avoir présenté une cartographie de la confusion qui permet de dégager les concepts périphériques à l'empire avec lesquels il est régulièrement confondu, nous identifierons donc trois travers dans les stratégies de définition dudit empire qui président à ce bruit sémantique. Révéler ces facteurs de confusion avec la grande puissance, l'hégémon, la cosmopolis et l'impérialisme, permet de proposer en conclusion un concept d'empire distinct. ${ }^{2}$ Nous y dégagerons les enjeux de cet effort de clarification et préciserons les perspectives qu'il ouvre pour l'analyse comparative des systèmes historiques mais aussi la compréhension de formes politiques contemporaines.

I Cette réflexion développe le travail entrepris avec Didier Chaudet et Florent Parmentier, qui a donné lieu à la publication de L'Empire au miroir. Stratégies de puissance aux États-Unis et en Russie aux éditions Droz en 2007, et qui s'est poursuivie avec la version enrichie: When Empire Meets Nationalism. Power Politics in the US and Russia, aux éditions Ashgate en 2009 ainsi qu'avec l'article «Circonscrire ce qui se déploie. Un essai de définition distinctive du concept d'empire» paru dans Commentationes Historiae luris Helveticae, IV, en 2009. Les références complètes de ces titres sont disponibles dans la bibliographie qui fait suite à cet article. L'auteur remercie Philippe Forget, Alice Goheneix, Till Hanisch, Jean Leca, Florent Parmentier, et les relecteurs de la Revue européenne de sciences sociales, qui ont bien voulu lire et critiquer des versions antérieures de ce texte.

2 Ce faisant, nous poursuivons l'effort entamé par Dario Battistella (2007). Nous nous confrontons ailleurs à partir du cas de l'empire romain à la seconde exigence classique de l'élaboration conceptuelle ailleurs: le souci de validité empirique du concept. Voir Pelopidas B. (2009). 


\section{CARTOGRAPHIE DE LA CONFUSION}

L'empire et ses concepts périphériques

Quiconque s'intéresse aux occurrences du terme «empire » constate aussitôt qu'il apparaît régulièrement lorsqu’on s'interroge sur la réalité, l'origine, les effets et l'éventuel déclin de la forme historique désignée par l'expression «empire américain » (voir notamment Wright Th., Nexon D. H., 2007). Difficile dans ce cas de distinguer l'empire de l'hégémon (Snyder, 2005) et de mesurer, si l'on exclut les rares partisans de l'«empire américain» qui acceptent cette appellation, en quoi il n'y a pas là une simple dénonciation d'un rapport au reste du monde que l'on peut qualifier d'impérialiste (Guerlain P., 2007 et Mann M, 2008). La décolonisation est alors pensée comme le processus inachevé structurant l'histoire récente. Ainsi, des enjeux mémoriels de qualification se retrouvent dans la réaffirmation des identités colonisées contre l'ancienne métropole qui réduisent l'empire à sa dimension coloniale et le désignent comme un oppresseur, voire un génocidaire (voir, concernant le cas français, l'exemple paradigmatique fourni par Oliver Le Cour Grandmaison, 2005). Reconnaissons que le champ de ce débat n'accorde guère de place au concept d'empire et se concentre sur celui d'impérialisme. ${ }^{3}$

De tels enjeux mémoriels se retrouvent dans les considérations sur le retour de la puissance russe, qualifiée d'empire en référence à l'ex-colosse soviétique qui lui-même refusait cette appellation, trop évidemment associée à la période tsariste. ${ }^{4}$ En dehors de l'usage politique de la référence au passé, le concept d'empire ne vaut ici que comme une variante assez mal distinguée de la notion de grande puissance ou de celle d'hégémon, entendue comme l'horizon d'un projet de restauration de l'influence russe sur l'ancien espace soviétique. ${ }^{5}$

3 Ainsi, en dépit de son titre et de son sous-titre, l'ouvrage d'Henry Laurens, L'Empire et ses ennemis. La question impériale dans l'histoire (2009) s'intéresse presque exclusivement à I'histoire des impérialismes et anti-impérialismes, surtout à partir du XIXe siècle.

4 Au-delà du rejet du passé tsariste, Terry Martin souligne que Lénine et Staline considéraient comme dangereux d'être désigné comme un empire à l'époque où fleurit le nationalisme: voir Terry Martin (200I, p. 19).

5 Pour une étude du projet de réaffirmation de la puissance russe sur la zone eurasiatique, ses origines intellectuelles et les pratiques de politique étrangère qui en découlent, articu- 
La taille de la Chine et les spéculations sur son ascension au rang de première puissance globale réhabilitent l'«Empire du milieu» (Salomon M., Doan Kêt V., 2006) mais le privent de l'expansionnisme communément associé au concept et oublient que l'attachement des Chinois à la notion de souveraineté révèle l'ancien colonisé plutôt qu'un quelconque empire. Il va sans dire que la Chine a été une très grande puissance que l'on a pu qualifier d'empire pendant une longue période de son histoire (voir Hucker Ch., 1995 [1975] et Chua A., 2007, chapitre 3). Toutefois, l'affirmation de la puissance chinoise après la mort de Mao ne repose pas seulement sur la mémoire d'un passé «impérial» issu d'une revalorisation de la civilisation confucéenne mais aussi sur la réaffirmation d'un sentiment d'humiliation à l'égard de l'Occident et du Japon au cours du siècle qui sépare la première guerre de l'opium (1842) et la capitulation du Japon dont les ambitions impériales visaient précisément la Chine. Ce sentiment est prégnant dans la population, surtout depuis les années 1990 (voir Gries, P., 2005 et Yang J. 2007, p. 264-275). Le concept d'empire, fréquemment mobilisé pour la désigner, peine donc à saisir l'entreprise d’autodéfinition de soi à laquelle s'adonne la Chine d’après Mao.

Ces usages, qui oscillent entre impérialisme, hégémon et grande puissance, ont en commun de considérer l'empire comme une forme politique.

Un usage plus large encore, fondé sur le simple sens de domination, le sens étymologique le plus élémentaire de l'empire, l'étend à la puissance économique japonaise, certes dirigée symboliquement par un empereur, mais aussi aux grandes entreprises et au monde de la finance.

La saisie de l'Union européenne à travers le prisme de l'empire combine plusieurs des éléments que nous avons aperçus. On y retrouve son intégration et sa puissance économique, l'opposition entre le «centre de gravité» de Joschka Fischer ou le «groupe pionnier» de Jacques Chirac et le reste des États de l’Union, renvoyant à la dichotomie centre-périphérie (Zielonka, J., 2006) ${ }^{6}$, mais

lée avec les concepts d'empire et d'hégémon, voir Didier Chaudet, Florent Parmentier et Benoît Pelopidas (2007, chapitres 2 et 5).

6 Pour une perspective critique sur l'UE comme un empire qui n'en assume pas la responsabilité, voir les travaux de David Chandler (2006). II faut aussi noter que l'application à l'URSS de la 
aussi son processus d'élargissement, sa politique de «voisinage » et son absence de frontière définitive à l'Est (Rupnik J., 2007). S'y ajoute parfois une forme de confusion entre empire et cosmopolis (voir, notamment, Beck U. et Grande E., 2007, qui évoquent explicitement la forme empire dans leur ouvrage dont le titre original, Kosmopolitisches Europa, suggère toutefois la «cosmopolis »).

On peut estimer qu'il n'y a rien de surprenant ou d'alarmant à ce que le débat politique mobilise des métaphores ${ }^{7}$, comme c'est le cas pour bien des usages du concept pointés ci-dessus. Toutefois, les universitaires ne parviennent qu'imparfaitement à réduire ce bruit sémantique (ou conceptual stretching, en anglais) qui étire et dissout la catégorie. Plusieurs motifs supplémentaires de confusion apparaissent ainsi dans les débats.

D’une part, certains entendent résoudre le débat sur la nature de la puissance américaine en évoquant un «empire à nul autre pareil ${ }^{8}$. Cela peut signifier deux choses: soit il s'agit d’un truisme suggérant que chaque expérience historique est unique, ce qui ne nous dit rien sur l'applicabilité de la catégorie d'empire à ce cas précis, soit cela veut dire que cette modalité de l'empire est

qualité d'empire conduit dans une perspective fonctionnaliste à faire de l'Union Européenne un «substitut d'empire»: voir Jacques Rupnik, (2003). Pour un aperçu historique des usages de l'idée d'empire appliqués à l'Union européenne, voir Jean-Luc Chabot (2006). Enfin, Herfried Münkler (2007) défend l'idée que l'Europe devra emprunter au modèle impérial dans l'avenir.

7 Une position radicale sur la question considérant que la pensée est impossible sans «métaphore conceptuelle» se lit chez George Lakoff et Mark Johnson, dans Les Métaphores dans la vie quotidienne, Paris, Minuit, 1986. Quoi qu'il en soit, accepter l'existence de métaphores dans le discours politique n'équivaut pas à négliger leurs effets. Pour un exemple des effets de la métaphore de la prolifération sur la compréhension et les réponses politiques au problème du nucléaire, voir Benoît Pelopidas, «Du fatalisme en matière de prolifération nucléaire. Retour sur une représentation opiniâtre», Revue suisse de science politique, vol. 15 n², été 2009, surtout p.286-29l et p.299-301.

8 "America's empire is like no empire of times past", peut-on lire sous la plume de Michael Ignatieff, dans l'article "The Burden" du New York Times Magazine en date du 5 janvier 2003. La même idée se retrouve dans "Is there an American Empire" de Michael Walzer (2003) mais aussi dans Andrew J. Bacevich (2002) Chalmers Johnson (2004) ou chez Ghassan Salamé (2005), pour lequel: «Si "empire" il y a, c'est dans une acception absolument nouvelle». Pour un dernier exemple récent, on pourra citer Georges Le Guelte (2009) qui écrit, à la page 257: «Les États-Unis sont à la tête d'un empire beaucoup plus vaste et plus puissant que tous ceux qui ont existé dans le passé». 
radicalement différente de toutes celles qui ont précédé, et que l'on suppose semblables entre elles. Si tel est le cas, pourquoi recourir à cette même catégorie?

D’autre part, l'empire désignerait la forme politique privilégiée des temps «ante-westphaliens », l'État territorialisé constituant au contraire le projet de la modernité westphalienne. Dans ce cadre, qui fait de l'empire la forme politique majoritaire d'un temps, on peut identifier deux positions, qui révèlent combien l'invocation de l'empire n'est qu'un accessoire qui redouble le débat sur la nature du temps présent et à venir, modernité ou post-modernité politique, actualité ou obsolescence de l'État westphalien couplé à la nation (voir Jordheim H., 2008). En effet, les deux positions extrêmes de ce débat sont les suivantes. Soit on considère que l'État souverain est «l'horizon indépassable de notre temps », comme le marxisme jadis, et l'empire s'avère une forme politique et une catégorie obsolètes ${ }^{9}$. Soit, on contraire, on envisage la mise en concurrence de la souveraineté et de la territorialité étatiques ${ }^{10}$. Dans ce cas, l'empire peut apparaître comme une catégorie séduisante pour désigner les nouvelles modalités de «gouvernance», puisqu'elle régissait le monde «ante-westphalien $»^{11}$. Cette tentation s'illustre particulièrement

9 «L'âge des empires, c'est l'histoire», affirme dans ce sens Joseph W. Esherick (2006, p. 385). Dario Battistella présente également le principe de souveraineté comme triomphant du principe imperial à la fin de la guerre de trente ans qui aboutit au traité de Westphalie dans Un monde unidimensionnel, 201 I, p. 30, 93-94. Certains critiques de l'ambition impériale américaine usent aussi de cet argument pour faire valoir que les États-Unis dans le monde contemporain n'en ont pas les moyens. Voir par exemple Michael Mann, qui écrit dans son Incoherent Empire, (2003, p. 97): «Le temps de l'empire est révolu» (notre traduction).

I0 Si l'on entend l'empire comme une forme politique plutôt qu'administrative, il faudrait le mettre en dialogue avec la nation. Nous ne cédons toutefois pas à la tentation de généraliser le projet français d'identification de l'État et de la nation, qui se retrouve dans l'idée wilsonienne du «droit des peuples à disposer d'eux-mêmes» alors que des États bi ou multinationaux existent. Pour un aperçu des configurations possibles, voir Alain Dieckhoff (2002) et les travaux de Juan Linz et Albert Stepan sur le couple «État-Nation et Nation-État» dont une formulation récente se lit sous la plume d'Albert Stepan (2008): "Do We Need a 'StateNation' Model as well as a 'Nation-State' Model'.

II À partir de l'exemple d'Israël, Bertrand Badie (1995, p. 19) soutient que «cet usage sélectif, par certains États, d'un modèle impérial [...] révèle déjà que le principe de territorialité qui s'est construit à l'ombre des États-nations est davantage un type idéal qu'une réalité concrète universelle. II suggère que tout ou partie de la logique territoriale de l'Empire peut émerger ou réémerger à tous moments et en tous lieux, en Europe ou ailleurs, dans un 
dans l'ouvrage de Jan Zielonka (2006) qui envisage l'Union européenne comme un «empire néo-médiéval ».12

Il faudrait ajouter deux positions significatives pour mesurer l'ampleur de la confusion à l'œuvre quant à la définition du terme «empire ». James Rosenau incarne la première. Il développe sa thèse sur la tendance à la fragmentation du pouvoir à l'échelle globale et à la démultiplication des centres de sorte que les individus en réseau deviennent les unités privilégiées de reconfiguration de l'ordre international. Ces éléments rapprocheraient a priori Rosenau de ceux qui remobilisent l'empire. Pourtant, il écrit explicitement que «l'histoire de l'empire s'est terminée» (Rosenau J., 2005, p. 74, notre traduction). La seconde position est tenue par les plus célèbres opposants contemporains à l'empire : Michael Hardt et Antonio Negri. Pour eux aussi, nous vivons une nouvelle «ère des empires » (Hobsbawm E., 1999 [1987]) ou plutôt de l'empire, et c'est là toute la différence. Cette ère correspond à un moment du capitalisme et de la globalisation marqué par le primat de la puissance américaine (voir Hardt M., Negri A., 2000 et Negri A, 2008). L'une des spécificités de leur approche tient précisément à ce que l'empire n'est pas la forme politique majoritaire à une époque mais qu'il désigne bel et bien le mode de gouvernement global propre à cette époque. La domination américaine n’en est qu’une composante, ce qui les distingue de la constellation qui recourt à l'empire pour récuser spécifiquement la politique étrangère des États-Unis. On mesure alors que même lorsque le diagnostic sur les tendances profondes qui affectent le monde contemporain est convergent, ses implications sur l'usage de l'empire sont imprévisibles. Cela s'explique par des définitions incompatibles, voire

contexte de crise». Estimer que la nation est la forme politique qui a succédé aux empires ne revient toutefois pas à affirmer que la mobilisation nationaliste est la cause unique et exclusive de la chute de ces derniers. Ainsi, la révolution russe est avant tout opposée à la guerre et secondairement à la dynastie des Romanov qu'à la forme impériale de la Russie. De même, en Chine, ce ne sont pas les indépendantistes du Tibet ou de Mongolie qui ont provoqué la Révolution de 1911 mais les Han; dans le cas du Mexique, la conscience nationale met plusieurs décennies à se former après la construction de l'État: voir Joseph W. Esherick, Hasan Kayali et Eric Van Young (2006, «Introduction»).

12 Voir également les discussions critiques en forme d'essai, foisonnantes et abrasives de Jean Leca (2009 et 20I0). 
radicalement opposées. Ainsi, certains tenants du post-national voient l'empire comme une forme de gouvernance fragmentée quand James Rosenau l'identifie à un pouvoir centralisé et monopoliste.

Alors que l'empire désigne pêle-mêle la grande puissance, l’hégémon, la cosmopolis ou une politique étrangère particulière qu'est l'impérialisme, voire qu'il ne s'applique qu'à un archaïsme qui n’a plus cours, l'effort de clarification exige dans un premier temps d'identifier les motifs de la confusion dans les stratégies de définition à l'œuvre.

\section{RUMEURS D'EMPIRE}

Les trois tentations de l'analyste

Parler d’empire, c'est hériter d’un terme à la généalogie millénaire, foisonnante et contradictoire, qui traverse les frontières et les langues.

Les traductions révèlent une première facette de la difficulté. À titre d'exemple, le terme grec signifiant «monarchie» sera traduit en latin par «empire ». ${ }^{13}$ Puisque le concept sera ici employé en français, la brève histoire que nous en proposons est elle aussi francophone. Apparu en II35 sous sa forme actuelle, issu du latin imperium, le terme désigne un «État soumis à l'autorité d'un empereur» et cette autorité elle-même. ${ }^{14}$ Depuis la première moitié du XII ${ }^{e}$ siècle, empire désigne par extension toute forme de pouvoir de quelqu'un sur quelque chose. À la même époque, il renvoie surtout à l'«autorité souveraine d'un chef d'État», la «force militaire», l'«armée» et la «réunion des vassaux ». À la fin du XVI ${ }^{\mathrm{e}}$ siècle, il s'applique à tout État ou groupe d'États doté d'un gouvernement central et, à partir du XVII ${ }^{\mathrm{e}}$, à un «État puissant et dominateur». Par métonymie, il désigne également le territoire où s'exerce la domination, voire la domination elle-même..$^{15}$ Une traversée de

13 On en trouve une trace dans les écrits du Pape Pie II (1458-|464) qui signale explicitement l'équivalence de termes. «Ce que les Grecs appellent monarchie, nous l'appelons empire», écrit-il (cité par Nederman C., 1993, p.510).

14 Ce sens s'appliquait déjà aux formes antérieures «empirie» (vers 1050) et «emperie» (vers ||20).

15 Voir Alain Rey, Dictionnaire historique de la langue française, dictionnaires Le Robert, Paris, 1995, 2 vol., vol.I, p.68I. 
l'histoire du terme «empire» permet d'identifier deux noyaux de sens: un pouvoir et son lieu d'exercice. ${ }^{16}$

Cette polysémie a eu une postérité que l'analyste contemporain ne peut ignorer. Ainsi, une tradition chrétienne fait de l'empire une figure métaphorique, l'instrument de la providence divine. Le récit, dit des quatre monarchies ou des quatre empires, apparaît explicitement dans une prophétie du livre de Daniel dans l'Ancien Testament (voir Muldoon J., I999, chapitre 5). Cette prophétie, qui n'est qu'un exemple, et sans doute pas le plus ancien, atteste de la postérité ambiguë du concept d'empire : elle a engendré des lectures tantôt providentielles ${ }^{17}$, tantôt apocalyptiques. Passons sur les débats relatifs à l'identification historique des quatre empires. Daniel n'en attend pas moins l'avènement du cinquième royaume, le royaume des cieux, qui succèdera aux quatre royaumes terrestres. Toutefois, saint Augustin redéfinit les quatre monarchies et conçoit ce cycle, qui culmine avec l'Empire romain, comme orienté vers l'avènement de l'Antéchrist. Le règne des cieux n’adviendra quaprès la chute de l'Empire. Sur le plan moral, on retrouve cette même ambigüité : la vision de Dante qui associe l'Empereur au souverain œuvrant pour la paix et l'accomplissement temporel de l'humanité dans son ensemble est largement renversée par la tradition de l'humanisme civique qui, à la Renaissance (XIV et $\mathrm{XV}^{\mathrm{e}}$ siècles), a conçu l'empire comme une forme de régime politique dégradé par rapport au précédent de la République romaine. Les républiques sont dès lors associées à la vertu et les empires à la corruption morale, selon la thèse de Hans Baron (1966). Les travaux de Quentin Skinner (1996 [1978]) et John Pocock (1997 [1975]), qui l'ont approfondie et amendée, montrent que les humanistes civiques faisaient partie d'un courant majoritaire de la pensée politique qui rejetait

16 Le mot latin Imperium signifiait originairement autorité ou domination légitime. Au cours de la période romaine, entre le premier siècle avant Jésus-Christ et le premier siècle après, le terme avait déjà connu un changement de sens. Voir J. S. Richardson, "Imperium Romanum: Empire and the Language of Power", repris dans David Armitage (1998). Pour une enquête plus approfondie sur le sens du terme à travers I'histoire et les formes politiques, voir Dominic Lieven (2000, p. 3-26).

17 L'Énéide de Virgile (29-19 av. J-C.) ou le livre II de la Monarchie de Dante (1310) sont deux autres exemples classiques de lectures de la vocation impériale romaine dans le sens de la Providence. Au Xvl $\mathrm{e}^{\mathrm{e}}$ siècle, le luthérien Johann Sleidan utilise encore la prophétie de Daniel pour défendre une croisade contre les Turcs dans son Quattuor summiis imperiis (I556). 
l'empire associé à la tyrannie. ${ }^{18}$ À l'issue de cette histoire foisonnante et contradictoire, l'empire pose trois grands problèmes à celui qui entend l'approcher : la postérité multiséculaire et contradictoire du concept débouche sur sa réduction à l'impérialisme, l’idée affichée que l'empire renvoie à une réalité insaisissable et enfin, l’analyse du phénomène impérial à une seule échelle. La définition qu’en donne Jean-Baptiste Duroselle, considérée comme classique, nous semble particulièrement significative parce qu'elle illustre plutôt qu'elle ne résout ces trois problèmes :

Aussi loin que nous remontions, nous trouvons des empires. Le temps, les lieux leur imposent des structures propres. Mais le phénomène est identique. La conquête insatiable, la soumission de peuples à la domination d'autres peuples, l'élargissement, l'imperium. (Duroselle J-B., p. 295.)

Reprenons-les de manière plus systématique.

\section{LA RÉDUCTION DE L'EMPIRE À L'IMPÉRIALISME}

Si l'on excepte les grands textes des Lumières opposés à l'empire, de Diderot à l'abbé Raynal en passant par les Réflexions sur la monarchie universelle de Montesquieu, un moment fort ${ }^{19}$ de disqualification de l'Empire commence au début du $\mathrm{Xx}^{\mathrm{e}}$ siècle avec la montée en puissance du marxisme-léninisme. ${ }^{20}$ L'empire est majoritairement perçu comme une forme particulière de domination sur ses sujets, et un mouvement historique de conquêtes territoriales.

I8 Cela se comprend dans le contexte de la lutte des cités du Regnum Italicum contre le Saint Empire romain germanique. Ces thèses sont aujourd'hui nuancées par Cary Nederman, qui retrouve la trace de certains humanistes de la Renaissance attachés à la figure de l'Empire, parmi lesquelles le futur Pape Pie II, qui avait occupé la fonction de secrétaire de Frédéric III (1440-1493). Ce tournant du sens de l'«empire» n'a pas été partagé partout. Le rêve d'un empire universel du Pape a demeuré jusqu'à la fin du Xvl siècle. Voir John Robertson, "Empire and Union: Two Concepts of the Early Modern European Political Order" dans John Robertson, (1995, p. 3-36) et Frances Yates (1985).

19 Entre temps, ces arguments n'ont pas disparu mais l'Empire a compté de nombreux partisans. Pour un aperçu des débats autour de la notion en France et en Grande Bretagne aux $X V I I{ }^{e}$ et $X I X^{e}$ siècles: voir Jennifer Pitts, 2005. En ce qui concerne la Révolution américaine: voir Norbert Killian, "New Wine in Old Skins? American Definitions of Empire and the Emergence of a New Concept" repris dans David Armitage (1998).

20 Rappelons que Marx n'a jamais vraiment élaboré une théorie de l'empire ou de l'impérialisme. 
Cette vision renvoie à une postérité majoritairement polémique du concept qui se traduit dans les débats contemporains.

Qui désigne l'empire accuse l'oppresseur, rappelant à demi-mot que l'imperium (le pouvoir sur les forces armées et le pouvoir par la force) n’est pas la potestas (le pouvoir imposé par la justice), pour reprendre les catégories romaines. Cette postérité va se cristalliser dans le concept d'impérialisme, qui prétend révéler la vérité de l'empire par une opération de réduction. Le concept d’impérialisme naît dans son acception moderne en anglais, à la fin du XIX siècle, sous la plume de l'économiste et essayiste américain Charles A. Conant (I898), comme recherche de nouveaux débouchés pour les capitaux ${ }^{21}$. Il est ensuite popularisé par l'étude de John Hobson: Imperialism, a Study (1902), à l'égard de qui Lénine reconnaît sa dette dans L’impérialisme, stade suprême du capitalisme (1916). Les deux études partagent une approche économique des trois dernières décennies du XIX siècle et surtout une acception accusatoire du concept, même si, chez Hobson, la réforme est à l'ordre du jour. ${ }^{22}$ À partir de sa captation par le vocabulaire marxiste-léniniste, «impérialisme» cristallise l'accusation. Vers 1954, il signifie : «domination morale, intellectuelle, psychique ${ }^{23}$ Les empreintes marxistes-léninistes et anti-colonialistes (voir Amin S., 1974) ${ }^{24}$ ont marqué le concept et sa connotation accusatoire s'est étendue à celui d'«empire». À partir de la dialectique entre le centre et la périphérie, le premier dominant la seconde, l'école dite de la Dependencia a combiné une critique globale du capitalisme et des visées anticoloniales. Raul Prebisch, André Gunder Frank, Immanuel Wallerstein, Samir Amin et Fernando Henrique Cardoso ont été les figures de proue de ce mouvement. Après la décolonisation, la forme culturelle de l'impérialisme restait à combattre. Les empires ne menaçaient plus les souverainetés mais ils visaient

21 En fait, les premiers emplois français du terme (en 1832) désignent la tendance favorable au régime napoléonien. Et Alain Rey date le vocable anglais "imperialism" de 1878: voir Dictionnaire historique de la langue française, dictionnaires Le Robert, p. I001-1002.

22 Pour une comparaison détaillée: voir A. M. Eckstein, "Is There a 'Hobson-Lenin Thesis' on Late Nineteenth Century Colonial Expansion?", The Economic History Review, 44 (2), 1991, p. 297-3I8.

23 Voir le Dictionnaire historique de la langue française, op.cit., p. I00 I-I002.

24 Pour une vue plus étendue sur les textes majeurs traitant de la notion d'impérialisme: voir P. J. Cain et M. Harrison (200I). 
toujours à façonner les cœurs et les esprits. L'un des travaux les plus significatifs de cette approche est sans doute Culture et impérialisme d’Edward Said (2000 [1993]), une lecture de la littérature anglo-saxonne du XIX ${ }^{\mathrm{e}}$ siècle considérée comme le symptôme d'une mentalité coloniale persistante, qui s'inscrit dans la continuité de son travail fondateur sur l'orientalisme. Les études postcoloniales suivent ce chemin et étudient par exemple les formes linguistiques de l'impérialisme. Le terme est alors un quasi-synonyme de colonialisme. Et l'impérialisme, à connotation exclusivement négative, demeure le destin de l'empire.

Ainsi, on retrouve cette idée derrière les stratégies rhétoriques des différents parties prenantes au débat sur la nature de la puissance américaine, tant en France qu’aux États-Unis.

Parmi les partisans américains de la suprématie de leur pays, très peu utilisent le terme d'«empire», du fait de l'identité de la République américaine comme ancienne colonie britannique qui s'est révoltée contre l'empire, certes, mais plus généralement du fait de la postérité du terme lui-même. Aussi n’emploient-ils pas le terme de manière isolée, mais l'associent-ils la plupart du temps à un adjectif laudatif, ${ }^{25}$ «libéral», «bienveillant» ou «lite $»^{26}$. D’ailleurs, après avoir écrit son article sur l'empire bienveillant, Robert Kagan n’a plus jamais usé du terme, auquel il préfère désormais celui d'«hégémonie ».27

25 Nous avons trouvé trois exceptions majeures: Max Boot, Sebastian Mallaby et Deepak Lal, avec leurs contributions respectives: "The Case for the American Empire. The most realistic response to terrorism is for America to embrace its imperial role", Weekly Standard, I5 October 200I, http://www.weeklystandard.com/Utilities/printer_preview.asp?idArticle=318, "The Reluctant Imperialist: Terrorism, Failed States, and the Case for American Empire", Foreign Affairs, 8 I (2), 2002, p.2-7 et In Praise of Empires, New York, Palgrave MacMillan, 2004. L'existence même de cette littérature suggère une évolution tendancielle de l'usage du concept aux Etats-Unis. Voir Helge Jordheim (2008).

26 Le chapitre 5 de l'ouvrage de Niall Ferguson (2004) s'intitule ainsi "The case for liberal empire"; Michael Ignatieff, a publié un Empire Lite, Nation Building in Bosnia, Kosovo, Afghanistan en 2003, et Robert Kagan son fameux article "The Benevolent Empire" en 1998. L'expression fameuse d'«Empire par invitation», popularisée par Geir Lundestad (1986 et 2003), illustre également ce phénomène bien qu'elle ait été forgée pour expliquer comment les Européens ont appelé une implication américaine dans leurs affaires après la Seconde Guerre mondiale.

27 Voir, par exemple, Kagan R. (2002). Dans son ouvrage sur les origines impérialistes de la nation américaine, Dangerous Nation: America's Place in the World from its earliest days to the Dawn of the $20^{\text {th }}$ Century (2006), il qualifie les pères fondateurs de «premiers impérialistes» 
Irving Kristol partage cette réticence et emploie de préférence imperium, «un terme plus subtil que celui d'empire » estime-t-il. ${ }^{28}$ Symétriquement, la postérité polémique du terme d'empire incite ceux qui voudraient disculper l'une ou l'autre forme politique à proposer une définition suffisamment exigeante pour qu'elle ne s'applique pas au cas d'espèce. Par exemple, en France, Alexandre Adler affirme que «l’Amérique n’a pas été, n’est pas, ne deviendra pas un Empire. » (2004, p. 28I) Il fonde sa thèse la définition suivante:

Pour qu'il y ait imperium, il faut un «pouvoir de glaive»- militaire au sens strict - qui s’exerce indépendamment de la loi intérieure - supra legem - sur des peuples dénués de droit. (Ibid.)

C'est l’une des définitions les plus exigeantes que nous ayons trouvée, inapplicable à quelque forme de domination contemporaine - ou passée - que ce soit pour peu que l'on reconnaisse à la coutume le statut de droit aux côtés de la loi.

À l'opposé, les pourfendeurs de l'empire vont faire un usage démultiplié du terme qui se traduit en général par deux attitudes. Ils débusqueront la marque de l'empire derrière la moindre forme d'influence ou l'investiront d'une dimension pathologique. L'extension extrême du concept d'empire chez Negri et Hardt, défini comme «une république universelle, un réseau de pouvoirs et de contre-pouvoirs structurés selon une architecture illimitée et inclusive » (2000, p. I68, notre traduction) incarne la première attitude. Or, si toute influence devient impériale, le qualificatif, lui, devient inutile. Le titre du deuxième chapitre de l'ouvrage de Chalmers Johnson, Nemesis: the Last Days of the American Republic (2007) illustre de manière éloquente la seconde : il aborde les “Comparative Imperial Pathologies : Rome, Britain and America”.

- c'est le titre du premier chapitre - mais semble prendre acte de la connotation du terme puisqu'il les définit par leur «désir agressif et apparemment insatiable de territoire et de domination.» p. 3, notre traduction.

28 Irving Kristol, “The Emerging American Imperium”, Wall Street Journal, 18 août 1997, section A p. 14. 
Après avoir observé la réduction polémique de l'empire à l'impérialisme qui brouille encore aujourd'hui les discours et les analyses et néglige la fécondité originaire du concept, il nous faut observer que la figure impériale survit à ses incarnations historiques successives. Cette pérennité alimente l'idée d'un objet proprement insaisissable, la seconde tentation de l'analyste.

\section{L'ILLUSION DE L'OBJET INSAISISSABLE ET LA TENTATION DE L'AUTO-DÉSIGNATION}

Cette idée d'un objet qui échapperait à toute définition ${ }^{29}$ masque des choix épistémologiques que les analystes refusent de faire ou n'explicitent pas. La définition de Duroselle citée plus haut entend restituer le mystère persistant par lequel le phénomène impérial échappe à la saisie des hommes et suscite en eux une fascination qui ressemble étonnamment au sentiment du sublime. L'empire, c'est la majesté et la démesure de l'histoire en marche, incarnées en une forme politique. C'est Hegel voyant «l'âme du monde» à cheval lorsque Napoléon passe sous ses fenêtres au lendemain de la bataille d’Iéna en I806. Porteur d'un mystère immémorial, le récit impérial révèle sa nature mythologique en tant que récit des origines et des destinées, en l'occurrence, trace du mystère des origines. C'est bien de mythologie qu'il s'agit puisque Duroselle, qui pourtant élabore une théorie à base d'histoire comme il l'annonce dans son introduction, fait remonter le phénomène impérial à des temps immémoriaux, qui échappent à la datation. Face à ce défi, on met souvent en avant une approche empirique et historique, qui entend identifier les manifestations concrètes de l'empire. Cette approche qui se veut sans a priori n'est pas tenable, car si l'on n'a pas idée de ce que l'on cherche, on ne pourra pas le reconnaître si on le trouve. Les tenants de cette approche s'en remettront la plupart du temps à l'«auto-désignation». Autrement dit, ils qualifieront d’empire toute forme politique ainsi nommée par elle-même et/ou ses adversaires. Cela conduit à accorder au Royaume de Bokassa le statut d'Empire mais à le refuser à l'Union soviétique et à Rome dont le seul titre, officieux, du temps d’Auguste était le Principat, comme le rappelle Claude Nicolet (1988, p. 27).

29 Certains analystes affirment qu'«une telle définition se révèlerait inutilisable». C'est le cas de Dominic Lieven (2000, p.417) qui s'accorde sur ce point avec Maurice Duverger (1980, p.6-7). 
À partir de là, les différents empires n'ont plus en commun qu'une appellation identique mais à la signification chaque fois différente, ou encore une dynamique réductrice qui renvoie à la postérité polémique de l'impérialisme. ${ }^{30}$ Les approches monographiques se parent ainsi des atours impériaux et n’accordent au concept qu'une valeur de simple ornement de l'ouvrage. L'approche typologique, la plus fréquente, qui distingue des modalités de l’imperium, repose également sur une confiance en l'auto-désignation ou sur une définition minimaliste de l'empire comme le simple exercice d'un pouvoir. ${ }^{11}$ L'empire devient non plus une réalité politique, mais presque une métaphore. ${ }^{32}$ Il nous reste à identifier un troisième et dernier biais.

\section{LA TENTATION D'UNE DÉFINITION UNIDIMENSIONNELLE}

Le dernier travers des observateurs de l'empire répond à leur prise de conscience du précédent. Mesurant le risque d'extension du concept ou de la définition impossible, ils choisissent au nom de la parcimonie un aspect et l'opérationnalisent. Paradoxalement, cette réduction unidimensionnelle qui entend limiter la confusion rapproche bien souvent l'empire de l'un des concepts périphériques que nous avons identifiés plus haut. Dans cette soussection, nous nous proposons donc d’illustrer ce résultat paradoxal des définitions unidimensionnelles de l'empire pour faire ressortir par contraste trois niveaux d'analyse qui nous semblent devoir être combinés de manière rigoureuse pour pouvoir dégager une figure distincte et féconde de l'empire.

30 Voir Alexander J. Motyl (1999, p. II6-1 I7) qui avait bien mis en garde les analystes contre ce biais.

31 Par exemple, Justin Vaïsse, dans son article sur «Les Sens de l'empire», paru en 2004, introduit en ces termes la typologie qu'il propose: "Comme "empire" est synonyme de "pouvoir", on peut établir une sorte de continuum, des formes historiques les plus dures d'exercice de ce pouvoir [...] à ses formes les plus douces [...].» Ce problème de la subdivision du concept, notamment pour faire face à des phénomènes antérieurement non pris en compte, s'est illustré dans les sciences humaines pour le concept de «démocratie». Voir, à ce sujet, David Collier et Steven Levitsky (1997, p. 430-45I).

32 II existe pourtant une alternative à l'auto-désignation, qui consiste à élaborer un noyau de sens propre au concept d'empire, grâce à l'établissement de critères distinctifs qui permettent d'élaborer une échelle de proximité des formes politiques historiques, qu'elles se soient baptisées empire ou pas, avec ce que nous entendons par là. Nous développons ce projet dans Pelopidas (2009). 
Ainsi, un premier niveau, statique, distingue l'empire par sa taille et la relation de subordination qui s'instaure entre le centre et la périphérie. Un deuxième niveau consiste en une analyse historique diachronique, qui s'attacherait aux modalités politiques de déploiement de l'empire dans l'espace et le temps. Enfin, un troisième niveau consiste à porter un autre regard sur cette dynamique historique, et à la considérer comme une dynamique historiale, au sens où l'empire porte l'histoire et accouche d'elle; il fait naître une possibilité historique. Insistons: la figure de l'empire ne peut être comprise de manière féconde qu'en incorporant ces trois aspects.

En effet, la confusion entre l'empire et la grande puissance peut provenir de la considération exclusive du premier niveau, statique. L’article récent de Paul McDonald illustre l'attrait persitant d'une définition unidimensionnelle fondée sur la domination, au nom précisément de la quête d'une définition parcimonieuse et opérationnalisable, qui évite la confusion pointée plus haut. Il explique ainsi que sa définition ne saisit pas l'empire comme «une unité politique cohérente, mais plutôt une stratégie pour organiser les relations internationales » (McDonald P. K., 2009, p. 82-83, notre traduction). C'est selon lui une stratégie possible pour un État fort vis-à-vis d'un autre État plus faible. Une telle définition évite effectivement d'étendre le concept mais elle rapproche considérablement l'empire de la grande puissance ou de la puissance régionale dans la mesure où elle manque l'un des problèmes fondamentaux qui fait l'intérêt de l'étude des empires: la spécificité de l'empire comme forme politique par rapport à l'État moderne.

De même, la réduction de l'empire à l'impérialisme peut se comprendre comme une insistance sur les deux premiers aspects, la suprématie et l'expansion, considérant le récit impérial comme un simple masque de la domination. Enfin, négliger le troisième aspect, la dimension intégrative, prophétique et révolutionnaire de l'empire peut conduire à le confondre avec un hégémon.

C'est pourquoi nous nous proposons de procéder à une anatomie de l'empire, persuadés que ce qui distingue l'empire réside dans la conjonction de ces trois aspects qu'il nous faut maintenant préciser : des caractéristiques politiques durables, une évolution dans le temps et l'espace mais aussi la conviction originaire partagée par ses sujets d’une vocation impériale révolutionnaire. 
Précisons la nature du projet qui sous-tend cette entreprise de définition. Elle entend proposer un concept distinct de ceux d'hégémon, de grande puissance, d'impérialisme, et sera amenée à se positionner par rapport à celui de cosmopolis. C'est ainsi que la finitude de l'empire, qui apparaît à première vue comme son destin propre, ne pourra être retenue. Que dit-elle au fond si ce n'est que toute forme politique est aussi historique? Elle naît, se déploie, décline et meurt. Les spécificités résident dans les modalités et le rythme du déploiement comme du déclin. Consciente de ce risque, notre approche distingue et explicite des noyaux de sens qui nous paraissent inhérents au concept d'empire et à l'aune desquels on pourra qualifier plus précisément les formes politiques étudiées. Si une approche typologique est retenue, elle pourra être précisée sur cette base et ne pas tomber dans le travers de l'auto-désignation. Ces noyaux de sens combinant les trois niveaux d'analyse que nous venons d'exposer - statique, diachronique, historial -, la définition qui en découle échappe également à la tentation unidimensionnelle et à ses travers.

\section{CONCLUSION}

Une définition multidimensionnelle de l'empire

En guise de conclusion, il nous faut revenir sur les enjeux qui fondent l'urgence d'un travail de définition distinctive de l'empire par rapport aux concepts connexes avant de proposer une définition qui réponde à ces exigences. Il ne sagit pas de dépassionner la rhétorique politique, ni de prétendre que ce soit possible, encore moins d'entrer en matière pour savoir si ce serait souhaitable. Néanmoins, sur le plan du débat académique, une définition distinctive de l'empire permettrait d'éviter les périls mentionnés de l'auto-désignation ou de la réduction de cette figure de la puissance à une seule de ses composantes. Un tel effort apparaît nécessaire à la poursuite féconde du débat sur l'historicité des empires. En effet, seule une définition distinctive et restrictive offrira une grille d'évaluation des discours tels que la fin de l'ère des empires, ou au contraire l'avènement d'un nouvel empire ou encore la qualification de l’Union européenne et de tout autre objet politique non-identifié à venir comme un empire. À l'issue de cet effort de clarification, nous proposons la définition distinctive suivante. 
L'empire se caractérise comme l'actualisation permanente par une communauté politique du récit de sa vocation historique; ladite communauté épouse la contrainte d'une expansion indéfinie de sa domination sur un territoire toujours plus vaste assimilé au tout du monde, auquel elle impose la paix et propose de s'associer au projet de transformation du monde qu'elle apporte..$^{33}$

Multidimensionnelle, elle ne cède ni à la réduction à l'impérialisme ni à la tentation de l'auto-désignation et, ce faisant, délimite un concept d'empire distinct de ceux de grande puissance, d’hégémon et de cosmopolis. ${ }^{34}$

\section{BIBLIOGRAPHIE}

Ouvrages

ADLER Alexandre, 2004, L'Odyssée Américaine, Paris, Grasset.

BACEVICH Andrew J., 2002, American Empire: The Realities and Consequences of U.S. Diplomacy, Cambridge, Harvard University Press.

BADIE Bertrand, 1995, La Fin des territoires, Paris, Fayard, 1995.

BARON Hans, 1966, The Crisis of the Early Italian Renaissance, Princeton, Princeton University Press.

BATTISTELLA Dario, 20II, Un monde unidimensionnel, Paris, Presses de Sciences Po.

BECK Ulrick et GRANDE Edgar, 2007 (2004), Pour un empire européen, trad. par Aurélie Duthoo, Paris, Flammarion.

CAIN Peter J., HARRISON Mark (dir.), 200I, Imperialism. Critical Concepts in Historical Studies, Londres, Routledge, 3 vols.

CHANDLER David, Empire in Denial: The Politics of State-Building, Londres, Pluto Press, 2006.

33 Cette définition a été préalablement avancée dans Chaudet, Parmentier, et Pélopidas (2007, p. 96).

34 Les étapes qui nous ont conduit à cette définition et sa mise à l'épreuve historique à partir du cas de l'empire romain sont détaillées dans Pelopidas (2009). 
CHAUDET Didier, PARMENTIER Florent et PELOPIDAS Benoît, 2007, L'Empire au miroir. Stratégies de puissance aux Etats-Unis et en Russie, Genève, Droz.

-, 2009, When Empire Meets Nationalism. Power Politics in the US and Russia, Aldershot, Ashgate.

CHUA Amy, 2007, Days of Empire, How Hyperpowers Rise to Global Dominance and Why They Fall, New York, Doubleday.

DIECKHOFF Alain, 2002, La Nation dans tous ses États, Paris, Flammarion.

DUROSELLE Jean-Baptiste, 1992, Tout empire périra, Paris, Armand Colin.

DUVERGER Maurice, 1980, (dir.) Le Concept d'empire, Paris, PUF.

FERGUSON Niall, 2004, Colossus. The Price of America's Empire, New York, Penguin Books.

HOBSBAWM Eric J., 1999 (1987), L'Ère des empires 1875-1914, traduit depuis l'anglais par J. Carnaud et J. Lahana, Paris, Hachette.

HUCKER Charles, 1995 (1975), China's Imperial Past, Palo Alto, Stanford University Press.

IGNATIEFF Michael, 2003, Empire Lite, Nation Building in Bosnia, Kosovo, Afghanistan, New York, Vintage Books.

JOHNSON Chalmers, 2004, Blowback: the Cost and Consequence of American Empire, New York, Henry Holt.

-, 2007, Nemesis: the Last Days of the American Republic, New York, Metropolitan Books.

KAGAN Robert, 2006, Dangerous Nation: America's Place in the World from its earliest days to the Dawn of the $20^{\text {th }}$ Century, New York, Alfred A. Knopf.

LAURENS Henry, 2009, L'Empire et ses ennemis. La question impériale dans l'histoire, Paris, Seuil.

LE COUR GRANDMAISON Olivier, 2005, Coloniser, exterminer: sur la guerre et l'Etat colonial, Paris, Fayard.

LE GUELTE Georges, 2009, Les Armes nucléaires. Mythes et réalités, Arles, Actes Sud.

LIEVEN Dominic, 2000, Empire, The Russian Empire and its Rivals, New Haven, Yale University Press. 
LUDESTAD Geir, 2003, The United States and Western Europe Since 1945. From "Empire" by Invitation to Transatlantic Drift, Oxford, Oxford University Press.

MANN Michael, 2003, Incoherent Empire, Londres, Verso, 2003.

MARTIN Terry, 200I, The Affirmative Action Empire: Nations and Nationalism in the Soviet Union, 1923-1939, Ithaca, Cornell University Press.

MOTYL Alexander J., 1999, Revolutions, Nations, Empires. Conceptual Limits,

Theoretical Possibilities, New York, Columbia University Press.

MULDOON James, 1999, Empire and Order, the Concept of Empire (800-1800), New York: St Martin's Press.

MÜNKLER Herfried, 2007, Empires, The Logic of World Domination from Ancien Rome to the United States, New York, Polity Press.

NEGRI Antonio, 2008, Empire and Beyond, New York, Polity Press.

- et HARDT Michael, 2000, Empire, Cambridge, Harvard University Press, trad. par Denis Armand Canal, Paris, Exil, 2000.

NICOLET Claude, 1988, L'Inventaire du monde: géographie et politique aux origines de l'empire romain, Paris, Fayard.

PITTS Jennifer, 2005, A Turn to Empire: The Rise of Liberal Imperialism in Britain and France, Princeton, Princeton University Press.

POCOCK John G. A., 1997 (1975), Le Moment machiavélien, traduction de Yves Lehmann, Paris, PUF.

ROBERTSON John (dir.), 1995, A Union for Empire: Political Thought and the British Union of 1707, Cambridge, Cambridge University Press.

SAID Edward W., 2000 (1993), Culture et Impérialisme, trad. par P. Chemla, Paris, Fayard.

SALAMÉ Ghassan, 2005, Quand l'Amérique refait le monde, Paris, Fayard, 2005.

SAMIR Amin, 1974, Accumulation on a World Scale; A Critique of the Theory of Underdevelopment, New York, Monthly Review Press.

SKINNER Quentin, 1996 (1978), Les Fondements de la pensée politique moderne, trad. par J. Grossman et al., Paris, Albin Michel. 
YATES Frances, 1985, Astrae. The Imperial Theme in the Sixteenth Century, London, Routledge and K. Paul.

ZIELONKA Jan, 2006, Europe as Empire, The Nature of the Enlarged European Union, Oxford, Oxford University Press.

\section{Articles}

BATTISTELLA Dario, 2007, «La notion d'empire en théorie des relations internationales», Questions internationales, 27, juillet-août, p. 27-32.

CHABOT Jean-Luc, 2006, «L'idée d'empire dans la représentation de la construction européenne» dans Thierry Ménissier (dir.), L'Idée d'empire dans la pensée politique, juridique et philosophique, Paris, L'Harmattan.

COLLIER David et MAHON James E. Jr, 1993, "Conceptual Stretching Revisited: Adapting Categories in Comparative Analysis", American Political Science Review, 87 (4), p. 845-855.

COLLIER David et LEVITSKY Steven, 1997, "Democracy with Adjectives: Conceptual Innovation in Comparative Research", World Politics, 49 (3), 1997, p. 430-45I.

CONANT Charles A., 1898, "The Economic Basis of Imperialism", North American Review, 502, p. 326-340.

JOSEPH W. Esherick, 2006, "The Return of Empire?" dans Joseph W. Esherick, Hasan Kayali et Eric Van Young, (dir.), From Empire to Nation. Historical Perspective on the Making of the Modern World, New York, Rowman \& Littlefield.

GRIES Peter, 2005, "Indignation, Nationalism and China's Japan Policy", SAIS Review of International Studies, vol.25, n², été-automne, p. I05-114.

GUERLAIN Pierre, 2007, «Le retour du discours impérialiste aux États-Unis», Revue française d'études américaines, 113, p.5-25.

JORDHEIM Helge, 2008,"Conceptual History between Chronos and Kairos: The Case of "Empire", Redescriptions. Yearbook of Political Thought and Conceptual History, II, p. ||5-|4|.

KAGAN Robert, 1998, "The Benevolent Empire", Foreign Policy, II I, p. 24-35.

-, 2002, "Power and Weakness", Policy Review, II3, 2002. 
KILLIAN Norbert, 1976, "New Wine in Old Skins? American Definitions of Empire and the Emergence of a New Concept" repris dans Erich Angermann et alii. (dir.), New Wine in Old Skins: A Comparative View of Socio-political Structures and Values Affecting the American Revolution dans David Armitage (dir.), 1998, Theories of Empire, 1450-1800, Aldershot, Ashgate.

LECA Jean, 2009, "The Empire Strikes Back!' An Uncanny View of the European Union. Part I - Do We Need a Theory of the European Union?", Government and Opposition, vol. 44 n³, juillet, p. 285-340.

-, 2010, "The Empire Strikes Back!' An Uncanny View of the European Union. Part II - Empire, Federation or What?", Government and Opposition, vol. 45 $n^{\circ} 2$, avril, p. 208-290.

LUDESTAD Geir, 1986, "Empire by Invitation? The United States and Western Europe, 1945-1952" Journal of Peace Research, 23 (3), p. 263-277.

MCDONALD Paul K., 2009, "Is Imperial Rule Obsolete. Assessing the Barriers to Overseas Adventurism", Security Studies vol. 18 nI, janvier, p. 79-1I4.

MANN Michael, 2008, «lmpérialisme économique et impérialisme militaire américains. Un renforcement mutuel?», Actes de la recherche en sciences sociales, 171-172, p. 20-39.

NEDERMAN Cary, 1993, "Humanism and Empire: Aenea Silvius Piccolomini, Cicero and the Imperial Ideal", The Historical Journal, 36.

PELOPIDAS Benoît, 2009, «Circonscrire ce qui se déploie. Un essai de définition distinctive du concept d'empire», Commentationes Historiae luris Helveticae, IV, p. I29-I44.

RICHARDSON J. S., 1991, "Imperium Romanum: Empire and the Language of Power", repris dans David Armitage (dir.), 1998, Theories of Empire, 14501800, Aldershot, Ashgate.

ROSENAU James, 2005, "The Illusion of Power and Empire", History and Theory, theme issue 44.

RUPNIK Jacques, 2003, «L'Europe centrale et les Balkans à la recherche d'un substitut d'empire» dans Anne-Marie Le Gloannec et Aleksander Smolar (dir.), Entre Kant et Kosovo. Études offertes à Pierre Hassner, Paris, Presses de Sciences Po, p. 339-360. 
- 2007, «quelles frontières pour l'Europe?» dans Jacques Rupnik, (dir), Les banlieues de l'Europe, Les politiques de voisinage de l'Union Européenne, Paris, Presses de Sciences Po, p.197-203.

SALOMON Matthieu et DOAN KÊT Vu, 2006, «L'Empire du Milieu perçu du Viêt-nam: grand frère incontournable et inquiétant», Outre Terre, 15 (2), p. 229-245.

SNYDER Jack, 2005, «Mythes d'empire et stratégie d'hégémonie», Critique internationale, 26, 2005, p. 59-78.

STEPAN Albert, 2008, "Comparative Theory and Political Practice: Do We Need a 'State-Nation' Model as well as a 'Nation-State' Model?", Government and Opposition, 43 (I), 2008, p. I-25.

VAÏSSE Justin, 2004, «Les sens de l'empire», dans Michel Wieviorka (dir.), L'Empire américain?, Paris, Balland, p. 201-212.

WALZER Michael, 2003, "Is there an American Empire", Dissent, 50 (4), p. 27-3I.

WRIGHT Thomas, Nexon Daniel H., 2007, "What is at Stake in the American Empire Debate?", American Journal of Political Science, I0I (2), 2007, p. 253-27I.

YANG Jian, 2007, "Of Interest and Distrust. Understanding China's Policy towards Japan", China: an International Journal vol.5 n², p. 250-275. 
